\title{
Effect of modern high-dose versus standard-dose radiation in definitive concurrent chemo-radiotherapy on outcome of esophageal squamous cell cancer: a meta-analysis
}

\author{
He-San Luo*, He-Cheng Huang and Lian-Xing Lin
}

\begin{abstract}
Background and objectives: Radiation Therapy Oncology Group (RTOG) 94-05 has demonstrated that higher dose radiation didn't improve outcome of patients with esophageal cancer (EC). However, several retrospective studies showed that a higher dose radiation based on modern radiotherapy techniques could improve overall survival (OS) and local control rate (LCR) of patients with EC, especially esophageal squamous cell cancer (ESCC). As trials have provided updated and controversial data, we performed this updated meta-analysis to investigate whether high-dose (> = 60 Gy) radiotherapy in definitive concurrent chemo-radiotherapy (CCRT) could yield benefit compared to standard dose radiotherapy.
\end{abstract}

Methods: A systematic literature search was carried out in the database of MEDLINE, PubMed and Embase. All studies published between 1 January 1990 and 31 December 2018 on the association between radiation dose and curative efficiency in EC were included in this meta-analysis. The hazard ratio (HR) was used to evaluate the time-to-event data employing RevMan version 5.3.

Results: Eight articles with a total of 3736 patients were finally included. Results indicated that there was a significant benefit in favor of high dose radiotherapy (HD-RT) regarding OS ( $H R=0.78,95 \% \mathrm{Cl}: 0.72-0.84, p<0.001$; 2-year OS risk ratio $(\mathrm{RR})=1.25,95 \% \mathrm{Cl}: 1.14-1.37, p<0.001)$, progression-free survival (PFS) $(P=0.001, \mathrm{HR}=0.7,95 \% \mathrm{Cl}: 0.57-0.87)$ and LRFS $(P<0.001, \mathrm{HR}=0.52,95 \% \mathrm{Cl}: 0.36-0.74)$.

Conclusions: HD-RT ( $>=60 \mathrm{~Gy}$ ) based on modern radiotherapy techniques in definitive CCRT appears to improve OS, PFS amd LRFS compared to the SD-RT in patients with ESCC.

Keywords: High-dose, Standard-dose, Chemo-radiotherapy, Esophageal squamous cell cancer, Meta-analysis

\section{Introduction}

Esophageal cancer (EC) is one of the most common malignant tumors and the fourth most common cause of cancer-related deaths worldwide [1, 2]. Approximately more than a half of the total cases occur in China [1]. In China, squamous cell cancer (SCC) is the most common type of EC, accounting for about $90 \%$ of all the patients

\footnotetext{
* Correspondence: Iuohesan@163.com

Department of Radiation Oncology, Shantou Central Hospital, Affiliated Shantou Hospital of Sun Yat-sen University, NO. 114 Waima Road, Shantou, Guangdong, China
}

(c) The Author(s). 2019 Open Access This article is distributed under the terms of the Creative Commons Attribution 4.0 International License (http://creativecommons.org/licenses/by/4.0/), which permits unrestricted use, distribution, and reproduction in any medium, provided you give appropriate credit to the original author(s) and the source, provide a link to the Creative Commons license, and indicate if changes were made. The Creative Commons Public Domain Dedication waiver (http://creativecommons.org/publicdomain/zero/1.0/) applies to the data made available in this article, unless otherwise stated.

with EC [3]. For early EC, surgery is the main curative treatment modality. However, most patients are not diagnosed until the disease is at an advanced stage [4].

For patients with locally advanced inoperable disease or patients refused surgery, definitive concurrent chemoradiotherapy (CCRT) is recommended as a standard treatment modality based on the results of the Intergroup Radiation Therapy Oncology Group (RTOG)8501 which improved the local control (LC) and overall survival (OS) with CCRT compared with radiotherapy (RT) alone [5]. The optimal radiation dose of CCRT was 
subsequently explored by the landmark RTOG 94-05 randomized controlled trial (RCT) and the interim analysis showed dose escalation from 50.4 to $64.8 \mathrm{~Gy}$ did not increase OS or local regional control [6]. Since then, 50.4 Gy has become the accepted standard dose for EC patients undergoing CCRT in Europe and American guidelines. Nevertheless, no consensus has been reached globally on the appropriate radiation dose of definitive CCRT for EC. More than a half of patients treated with standard-dose CRT were eventually developed recurrence or distant metastases and succumbed to this disease [7]. A recent systematic review has performed a pooled analysis to investigate whether high-dose radiotherapy (HD-RT) could improve LC or OS and found that HD-RT improved clinical outcomes as compared to standard-dose radiotherapy (SD-RT) [8]. As several studies have indicated that a higher dose above 50.4Gy of CCRT could be safely administered without significant untoward effects and yield high probability of LC [9-11], a dose of $60.0 \mathrm{~Gy}$ or more has become a more popular dose of CCRT in Asian countries, where ESCC is the predominant histological type. However, There is no prospective clinical trial to investigate the role of HD$\mathrm{RT}$ based on modern radiation techniques on the prognosis of ESCC patients.

In this study, we performed an updated meta-analysis to evaluate whether a higher dose above 60Gy of CCRT could improve the prognosis of ESCC patients as compared to a standard dose.

\section{Patients and methods}

\section{Search strategy, studies identification and selection}

A systematic literature search was carried out in the database of MEDLINE, PubMed and Embase. All studies published between 1 January 1990 and 31 December 2018 on the association between radiation dose and curative efficiency in EC were considered in this metaanalysis. The following terms were used for search: ("esophageal"[Title]) or ("oesophageal"[Title]) or ("esophagus"[Title])) and ("tumor"[Title]) or ("cancer"[Title]) or ("carcinoma"[Title]) or ("neoplasm"[Title]) or ("neoplasms"[Title])) and ("chemoradiation"[Title]) or ("chemoradiotherapy"[Title]) or ("radiochemotherapy"[Title]) or ("chemo-irradiation"[Title]) or ("chemo-radiotherapy"[Title])) and ("dose" [Abstract]). Inclusion criteria were defined as follows: 1) Studies on patients with esophageal cancer treated with concurrent chemoradiotherapy. 2) Studies comparing the curative efficiency in EC patients who were treated with high dose radiotherapy (HD-RT) or standard dose radiotherapy (SD-RT) (HD-RT was considered $>=60 \mathrm{~Gy}$ of radiation dose and SD-RT was considered 45-59.4 Gy of radiation dose). 3) Studies included most patients with esophageal squamous cell carcinoma. Studies were excluded as following: 1)
Neoadjuvant or adjuvant chemoradiotherapy combined with surgery. 2) Radiotherapy was delivered by Co- 60 or by unconventional fractions. 3) Review or case report, with other sites of cancers, and meta-analysis. 4) Results mixed with HD-RT and SD-RT, results not reported exactly. The search did not restrict the type of publication or periodical, but limit to English language.

\section{Quality assessment}

The quality of included studies was independently assessed by two assessors. The 9-star Newcastle-Ottawa Scale (Available from: http://www.ohri.ca/programs/clinical_epidemiology/oxford.htm) was adopted for assessment of the non-randomized studies. The quality categories were defined as follows: high quality (score 7-9), medium quality (score 4-6) and low quality (score less than 4). Quality of RCT was assessedusing the 7-point JADAD scale.

\section{Endpoints of interest}

We collected the information about OS, 2-year survival rate, progression-free survival (PFS) and local recurrencefree survival (LRFS) from the included studies. Patients were divided into two groups according radiation dose threshold as defined by the individual studies.

\section{Data extraction and synthesis}

The abstract of each study was reviewed by HS Luo and irrelevant or overlapping studies were removed according to the criteria mentioned above, thus creating a preliminary set of potentially relevant publications (Fig. 1). Then, two authors (HC Huang and LX Lin) independently reviewed the full articles to exclude studies unqualified and extracted data from all included studies regarding the first author, country, year of publication, study period, type of study, sample size, age, clinical stage, pathological types, chemotherapy regimens, radiation technology, and radiation dose in HD-RT and SDRT groups. The evaluation results were compared and re-evaluated until consensuses were reached between two authors. The frequencies of LRFS and PFS from the different groups were expressed as an OR with its 95\% CI. If a figure for $\mathrm{HR}$ and $95 \% \mathrm{CI}$ was not available, an estimate value was calculated indirectly by using the methods described by Tierney et al. Survival rates from Kaplan-Meier curves were read using Engauge Digitizer version 4.1 (available from: http://digitizer.sourceforge. net/) and the resulting data were then entered in the calculation spreadsheet appended to Tierney's paper.

\section{Statistical analysis}

All analyses were performed using Review Manager Version 5.3. Statistical heterogeneity among various studies was tested using I2-statistic. Fixed-effects model was used for risk ratio (RR) and hazard ratio (HR) analysis if 


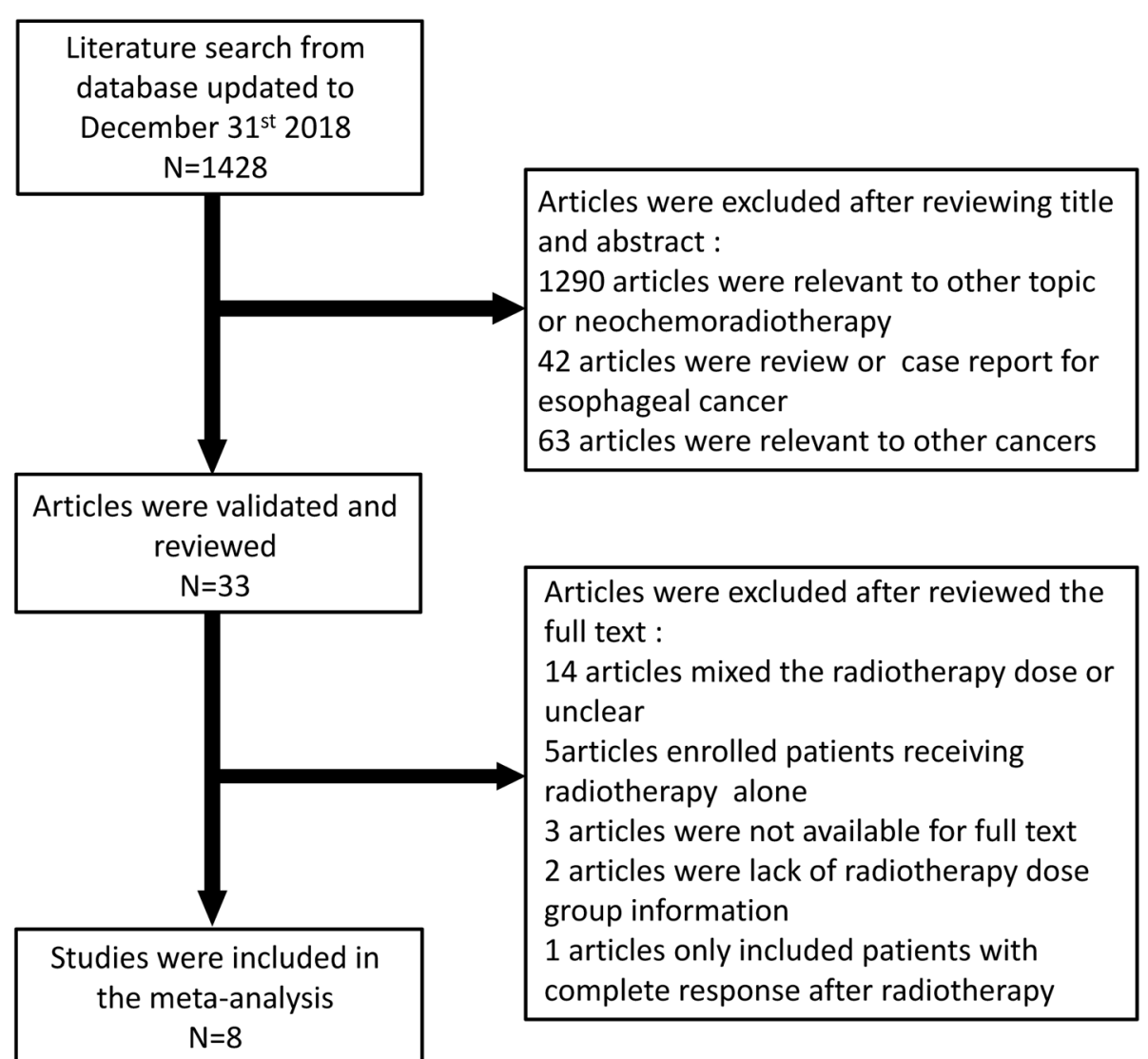

Fig. 1 Literature search strategy and study selection for the meta-analysis

there was no statistical heterogeneity (I $<50 \%, P \geq 0.1$ ) among studies, otherwise random-effects model would be used. Forest plots were generated to show the estimated RRs and HRs, representing the theoretical gain in absolute percentage on the basis of OS, 2-year survival rate, LRFS, and PFS in the included trials. Upper limit and lower limit of $95 \%$ confidence intervals (CIs) were calculated. The tests were considered statistically significant if $P$ values were less than 0.05 . All the $P$ values were two sided. Funnel plots were used to assess the potential of publication bias.

\section{Grading of Recommendations Assessment, Development and Evaluation (GRADE) assessment}

We followed the GRADE approach to assess the quality of the evidence from this meta-analysis. In this approach, guidelines apply a set of predetermined domains that either increase or decrease the level of confidence in the evidence. Domains that reduce confidence in the evidence are: risk of bias, inconsistency of results, indirectness of evidence, imprecision, and publication bias. On the other hand, a large magnitude of effect, confounding that increases effect magnitude and a dose-response gradient can increase confidence. Two researchers discussed the domains for each outcome until consensus was reached.

\section{Results}

\section{Eligible studies}

We identified 1428 potential relevant articles from electronic databases according the defined search strategy. After layer of screening by examination of the titles, abstracts, and the full texts, 1420 articles were excluded according the inclusion criteria and exclusion criteria. As a result, eight articles, with a total of 3736 patients, were included in the final meta-analysis (Fig. 1).

\section{Characteristics of included studies}

The detailed characteristics of these included studies were summarized in Table $1[6,12-18]$. One RCT (RTOG 94-05) and seven retrospective studies comprised the population of the meta-analysis. There were six studies from Asian country (including two from Korea, one from Taiwan area and three from China) and two studies from western country (including one from USA and one from France). All the patients were treated 


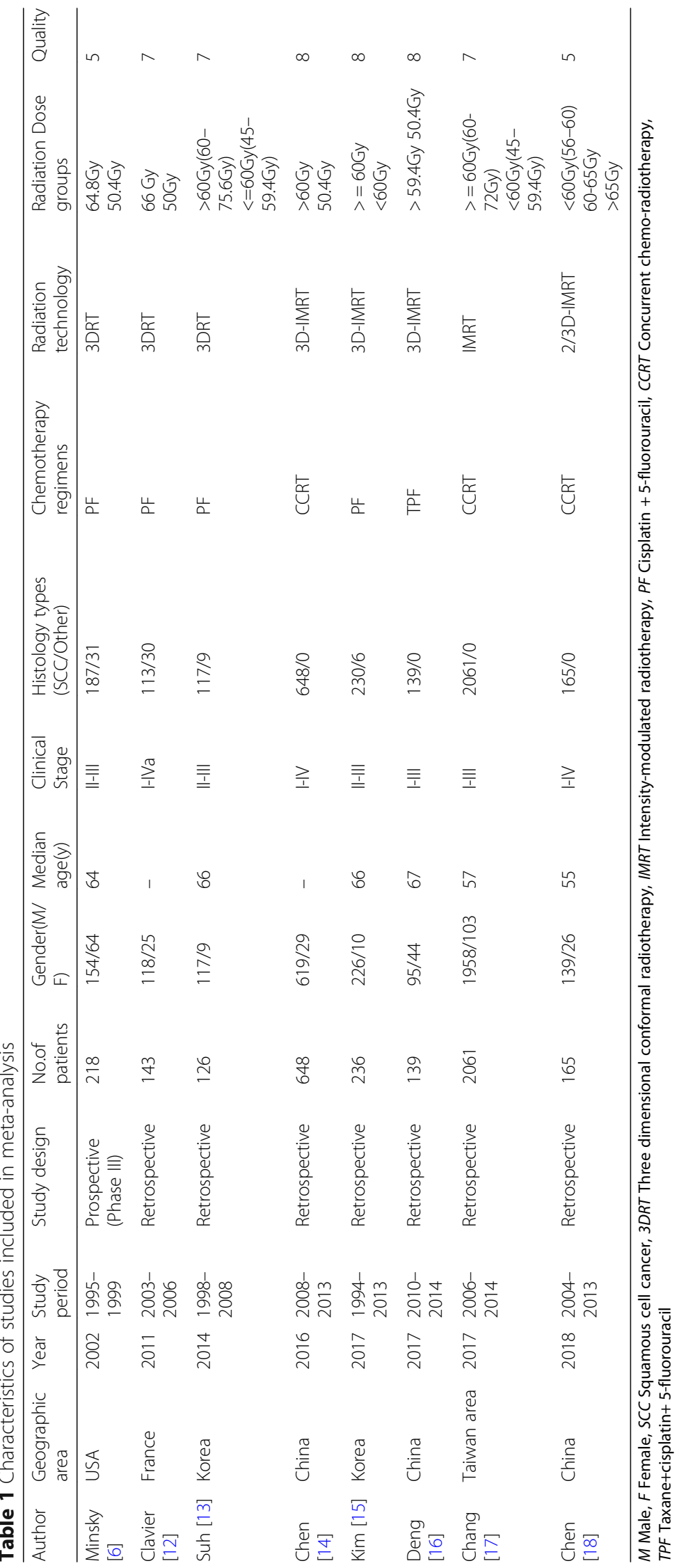


with concurrent chemo-radiotherapy. The majority of the enrolled patients were pathologically diagnosed as esophageal squamous cell carcinoma (3660/3736, 98.0\%). Most of the patients received modern radiotherapy (3Dconformal RT or IMRT). The total delivered radiation dose ranged from 45 to $75.6 \mathrm{~Gy}$. All eight studies provided information on overall survival. Four studies reported the PFS and two studies reported LRFS. HR and its 95\% CI were estimated based on the KaplanMeier curves of patients receiving the assigned different dose of radiation. The thresholds between HD-RT and SD-RT from each study were mainly around $60 \mathrm{~Gy}$.

\section{Effect of radiation dose on overall survival}

Eight articles reported overall survival curve of patients in HD-RT and SD-RT groups. There was no significant heterogeneity for the results among these studies $(P=$ $0.38, \mathrm{I} 2=6 \%$ ) that a fixed-effects model was used for further analysis. As shown in Fig. 2, patients in HD-RT group had overall survival benefit when compared with patients in SD-RT group (pooled $\mathrm{HR}=0.78,95 \% \mathrm{CI}$ : $0.72-0.84, p<0.001$ ). In addition, as shown in Fig. 3, benefit in 2-year overall survival rate was gained by patients in HD-RT group (pooled $\mathrm{RR}=1.25,95 \% \mathrm{CI}$ : 1.14 1.37, $p<0.001$ ). A fix-effects model was applied in this analysis because of no significant heterogeneity among these studies $(P=0.07, \mathrm{I} 2=47 \%)$.

\section{Effect of radiation dose on PFS}

PFS data was extracted from four studies including 594 patients. There was no significant heterogeneity among these studies $(P=0.27, \mathrm{I} 2=23 \%)$, hence a fixed-effects model was used for pooled analysis. As shown in Fig. 4, PFS of patients in HD-RT group was significantly better than that in SD-RT $(P=0.001, \mathrm{HR}=0.7,95 \% \mathrm{CI}$ : $0.57-0.87)$.

\section{Effect of radiation dose on LRFS}

LRFS data was reported in two studies including $362 \mathrm{pa-}$ tients. There was no significant heterogeneity between the two studies $(P=0.22$, I $2=34 \%)$, hence a fixed-effects model was used for pooled analysis. As shown in Fig. 5, LRFS of patients in HD-RT group was significantly better than that in SD-RT $(P<0.001, \mathrm{HR}=0.52,95 \% \mathrm{CI}$ : $0.36-0.74)$.

\section{Publication bias and GRADE assessment}

Publication bias statistical analysis was performed using the Funnel Plot. As shown in Fig. 6, no publication bias was detected in meta-analysis of HD-RT vs SD-RT ( $P=$ $0.38, \mathrm{I} 2=6 \%$ ).

A summary of findings table is presented in Additional file 1. We used the GRADE approach for this meta-analysis to appraise the confidence in estimates. In line with GRADE guidelines, the non-randomized studies started as low quality due to residual confounding. Furthermore, publication bias, an overall large effect and a dose response gradient were not identified. Thus, the studies were deemed to be of low quality.

\section{Discussion}

Definitive CCRT is considered as the optimal choice for patients with non-operable EC, especially ESCC. However, the standard dose of RT still remains controversial. On the basis of results from RTOG 94-05, 50.4 Gy has been accepted as standard dose in western countries and recommended by NCCN guideline for more than a decade [6]. Based on the theory of radiation biology, 50.4Gy is just adequate to control microscopic cancer cell, but inadequate to control a gross tumor lesion [19]. A radiation dose more than $60 \mathrm{~Gy}$ or even nearly $100 \mathrm{~Gy}$ is required to control and cure a gross solid tumor [19]. According to statistics, only a few patients with EC received a radiation dose of 50.4Gy would achieve

\begin{tabular}{|c|c|c|c|c|c|c|}
\hline Study or Subgroup & log[Hazard Ratio] & SE & Weight & $\begin{array}{l}\text { Hazard Ratio } \\
\text { IV. Fixed. 95\% Cl }\end{array}$ & $\begin{array}{r}\text { Hazard } \\
\text { IV. Fixed }\end{array}$ & $\begin{array}{l}\text { d Ratio } \\
\text { d. } 95 \% \mathrm{Cl}\end{array}$ \\
\hline Bruce2002 & -0.3147 & 0.1931 & $3.9 \%$ & $0.73[0.50,1.07]$ & 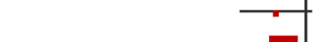 & \\
\hline Chang2017 & -0.2357 & 0.0473 & $65.4 \%$ & $0.79[0.72,0.87]$ & $\mathbf{a}$ & \\
\hline Chen2016 & -0.2744 & 0.0957 & $16.0 \%$ & $0.76[0.63,0.92]$ & - & \\
\hline Chen2018 & -0.9676 & 0.3537 & $1.2 \%$ & $0.38[0.19,0.76]$ & - & \\
\hline Clavier2011 & 0.0953 & 0.2162 & $3.1 \%$ & $1.10[0.72,1.68]$ & - & \\
\hline Deng2017 & -0.3857 & 0.2106 & $3.3 \%$ & $0.68[0.45,1.03]$ & $=$ & \\
\hline Hyun2017 & -0.2485 & 0.1783 & $4.6 \%$ & $0.78[0.55,1.11]$ & $\longrightarrow$ & \\
\hline Yang2014 & -0.3285 & 0.2398 & $2.5 \%$ & $0.72[0.45,1.15]$ & & \\
\hline Total $(95 \% \mathrm{Cl})$ & & & $100.0 \%$ & $0.78[0.72,0.84]$ & $\checkmark$ & \\
\hline \multicolumn{4}{|c|}{$\begin{array}{l}\text { Total }(95 \% \mathrm{Cl}) \\
\text { Heterogeneity: Chi }{ }^{2}=7.46, \mathrm{df}=7(\mathrm{P}=0.38) ;\left.\right|^{2}=6 \% \\
\text { Test for overall effect: } Z=6.57(\mathrm{P}<0.00001)\end{array}$} & & $\begin{array}{lcc}0.05 & 0.2 & 1 \\
& \text { Favours [HD-RT] }\end{array}$ & $\begin{array}{c}1 \\
\text { Favours [SD-RT] }\end{array}$ \\
\hline
\end{tabular}




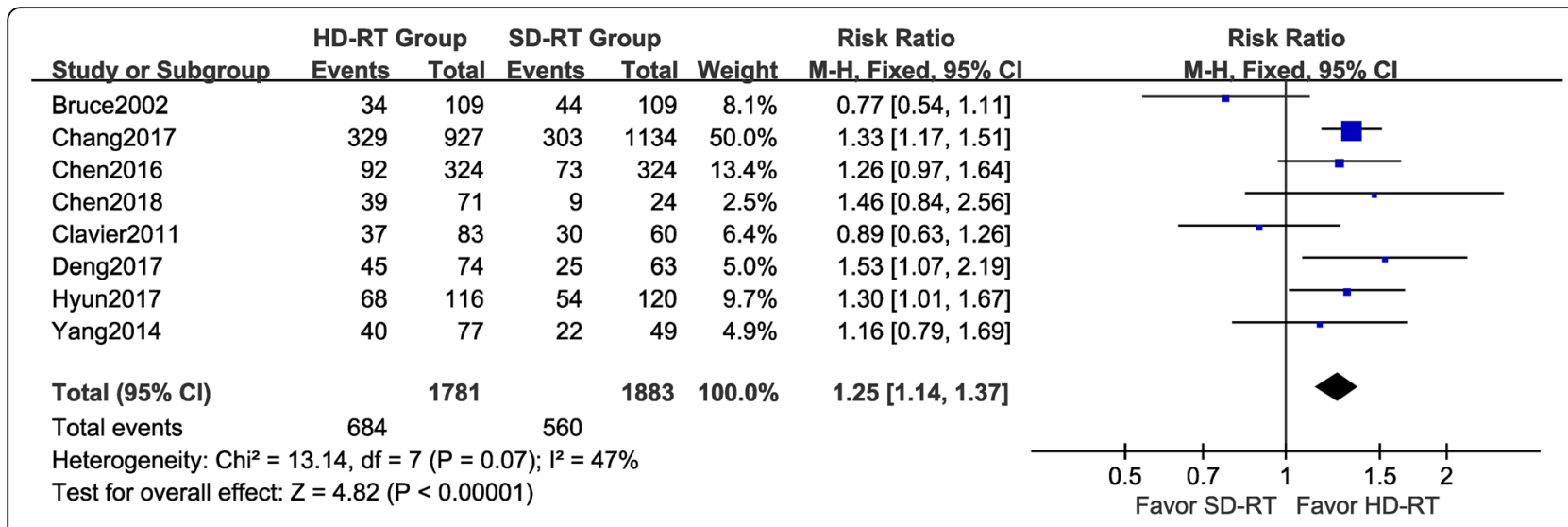

Fig. 3 Forest plot of risk ratio (RR) of 2-year overall survival (OS) rate between high dose radiotherapy (HD-RT) and standard dose radiotherapy (SD-RT) in EC patients treated with chemo-radiotherapy

complete response and obtain a long term survival [20]. Although RTOG 94-05failed to show evidence of increased dose could improve LC and OS, several retrospective studies have reported that increased radiation dose could improve local control and OS by enhancing clinical complete response rate. A pooled analysis from Song et al. showed that a higher radiation dose could improve clinical outcomes without significantly increasing radiation-related toxicities, which was contradictory to RTOG 94-05 [8]. However, this review was a compilation of single treatment arm, with only 3 included studies containing both a HD-RT ( $\geq 60 \mathrm{~Gy}$ ) arm and SD-RT arm. Another meta-analysis has indicated that patients who received $\geq 60$ Gy radiation had a significantly better prognosis as compared with $<60$ Gy [21]. The studies including patients received $<50$ Gy radiation and the studies including patients diagnosed as esophageal adenocarcinoma were included in the meta-analysis. In this present study, we selected studies which contained both HD-RT and LD-RT arms and studies in which the patients received radiotherapy delivered by modern radiotherapy technique such as 3D-RT and IMRT to reduce bias and heterogeneity. We excluded studies those mainly included patients with esophageal adenocarcinoma and patients received $<45$ Gy radiation. Our study indicated that HD-RT improve OS, PFS and LRFS as compared to SD-RT. With this meta-analysis, we supported that patients with non-operable ESCC should be treated with a higher dose radiation of $\geq 60$ Gy in clinical practice, as well as hoped that further randomized control trial comparing HD-RT with SD-RT delivered by modern radiation techniques in specific ESCC population would be carried out in our country.

In RTOG 94-05 and previous retrospective studies, RT was delivered by 2DRT or Co60 radiation which was now-outdated technique and may bring about risk of radiation toxicity in lung and heart $[6,22]$. With the clinical application of more precise radiation techniques such as 3DRT or IMRT, interpretation about the results of RTOG 94-05 should be different. 3DRT and IMRT might provide radiation dose escalation to the gross tumor volume while reducing the dose to the organs at risk [23]. In the RTOG0436 trial, a V20 limit of $<25 \%$ would reduce the incidence of grade 3-4 dyspnea with only $1.6 \%(5 / 319)$ in the CCRT arm with 3D-CRT [24]. Hsieh et al. reported that no patient suffered from symptomatic pneumonitis using IMRT to a total dose $>50.4$ Gy in a series of 29 patients with locally advanced EC

\begin{tabular}{|c|c|c|c|c|c|c|c|}
\hline Study or Subgroup & log[Hazard Ratio] & $\mathrm{SE}$ & Weight & $\begin{array}{l}\text { Hazard Ratio } \\
\text { IV. Fixed, } 95 \% \mathrm{CI}\end{array}$ & $\begin{array}{r}\text { Hazard } \\
\text { IV, Fixed }\end{array}$ & $\begin{array}{l}\text { d Ratio } \\
\text { d. } 95 \% \mathrm{Cl}\end{array}$ & \\
\hline Chen2018 & -0.6733 & 0.3059 & $12.4 \%$ & $0.51[0.28,0.93]$ & & & \\
\hline Deng2017 & -0.3285 & 0.2069 & $27.1 \%$ & $0.72[0.48,1.08]$ & & & \\
\hline Hyun2017 & -0.1625 & 0.161 & $44.7 \%$ & $0.85[0.62,1.17]$ & & & \\
\hline Yang2014 & -0.6733 & 0.2707 & $15.8 \%$ & $0.51[0.30,0.87]$ & $\pi$ & & \\
\hline Total $(95 \% \mathrm{Cl})$ & & & $100.0 \%$ & $0.70[0.57,0.87]$ & $\nabla$ & & \\
\hline \multicolumn{4}{|c|}{$\begin{array}{l}\text { Heterogeneity: } \mathrm{Chi}^{2}=3.91, \mathrm{df}=3(P=0.27) ;\left.\right|^{2}=23 \% \\
\text { Test for overall effect: } Z=3.27(P=0.001)\end{array}$} & & $\begin{array}{ccc}0.02 & 0.1 & 1 \\
& \text { Favours [HD-RT] }\end{array}$ & 1 Favours [s & $\begin{array}{c}10 \\
\text { SD-RT] }\end{array}$ \\
\hline
\end{tabular}

Fig. 4 Forest plot of hazard ratio of progression-free survival (PFS) between high dose radiotherapy (HD-RT) and standard dose radiotherapy (SD-RT) in EC patients treated with chemo-radiotherapy 


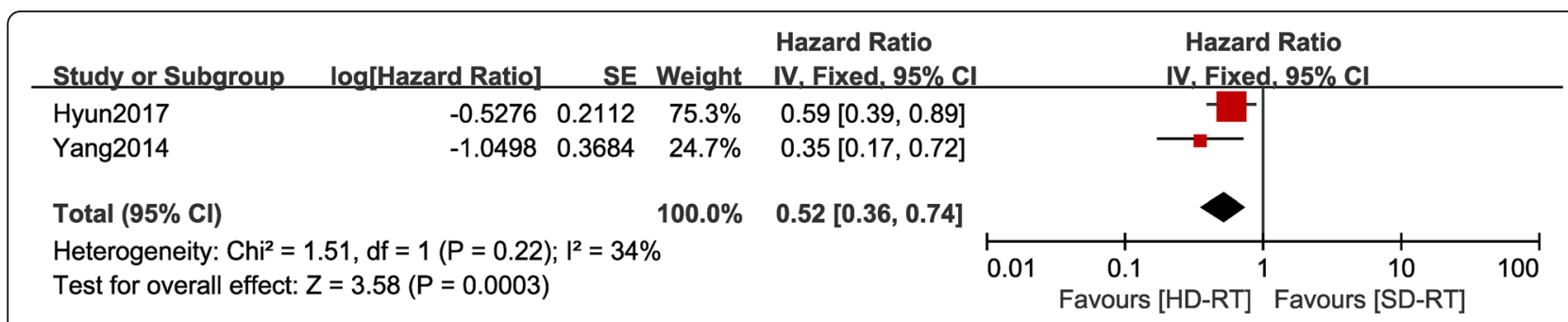

Fig. 5 Forest plot of hazard ratio of local recurrence-free survival (LRFS) between high dose radiotherapy (HD-RT) and standard dose radiotherapy (SD-RT) in EC patients treated with chemo-radiotherapy

[25]. These modern techniques allow the radiation oncologist to deliver higher doses of radiation with less toxicity to the surrounding normal tissue, prompting oncologist to investigate whether dose escalation based on modern radiation techniques improve patients outcomes safely, especially for ESCC patients. A phase II study from Chen and his colleague have demonstrated that radiation dose escalation using simultaneous modulated accelerated radiotherapy (SMART) combined with concurrent chemotherapy was feasible in ESCC patients with tolerable acute toxicities [11]. However, there is lack of a randomized control trial to investigate whether higher radiation dose using modern radiation technique improve outcome of ESCC patients with tolerable toxicities. In this study, we provided an up-to-date, reliable, and comprehensive summary of the effect of HD-RT compared to SD-RT in patients received 3DRT and IMRT, which would give us some implications for clinical practice and future research.

According to radiation biological theory, a radiation dose of 60 Gy or higher is needed to abrogate a gross solid tumor [19]. Therefore, 60Gy was frequently used as a threshold between high dose and standard dose in many studies when investigating the effect of radiation dose escalation in non-operable EC patients [13, 26]. However, in some studies, especially studies from western countries, one value between 50Gy and 60Gy was used as the threshold value for high dose and standard dose, which would bring about dose heterogeneity when a meta-analysis was performed [27, 28]. In this present study, we defined a radiation dose of $>=60 \mathrm{~Gy}$ as HDRT and $<60$ Gy as SD-RT. Furthermore, only those studies using 60Gy as a threshold value for high dose and standard dose were included in our meta-analysis, and we have found that high radiation dose of $>=60$ Gy was associated with better OS, PFS and LRFS, which is consistent with Chen's study and Song's study [8, 21]. However, how high radiation dose is appropriate for ESCC patients? According to Chen's study, a dose of $\geq 60$ Gy could improve patients' OS compared with a dose of < $60 \mathrm{~Gy}$, but an extremely high radiation dose of $70 \mathrm{~Gy}$ did not result in extra benefit or clinical outcome. In a Phase II dose-escalation study, Chen et al. indicated that a simultaneous integrated boost radiotherapy (RT consisted of 66 Gy at 2.2 Gy per fraction to the gross tumor) combined with concurrent chemotherapy is feasible in

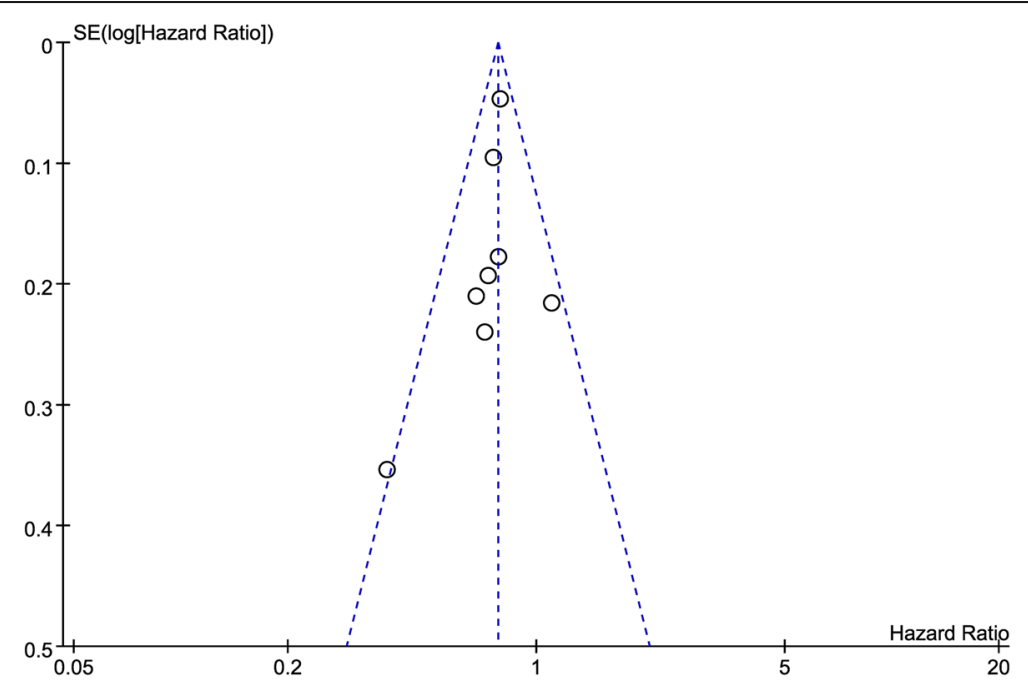

Fig. 6 Funnel Plot for publication bias of selected meta-analyses 
EC patients with tolerable acute toxicities [11]. In their study, only 5 of them $(8.4 \%)$ had local recurrence, while there were already 2 cases $(3.3 \%)$ of treatment-related death due to esophageal hemorrhage. Another recently published Phase I dose-escalation study, Yu et al. suggest that it is feasible to deliver up to $70 \mathrm{~Gy}(2.8 \mathrm{~Gy} / \mathrm{F})$ to the GTV [10]. However, no information of late toxicities was provided in this study. Thus, a radiation dose between 60Gy and 70Gy may be optimal and further dose-escalation may lead to increased risk of treatmentrelated death, rather than clinical benefit.

Inevitably, there are some limitations in this study. Firstly, most of the studies included were retrospective studies except one RCT and one population based propensity-score matched analysis. Secondly, several studies had a relative small amount of patients in each group. Thirdly, there were significant heterogeneities in PFS and LRFS meta-analysis. Fourthly, some specific information, such as chemotherapy regimens and radiotherapy field design, were unable to be obtained in the included studies, hence we could not conduct subgroup analyses based on these factors. Last but not the least, due to non-randomized approach to treatment selection, there must be some potential unmeasured selection biases regarding the patient physical and organ status, same clinical stage of disease but more extensive disease, preference of radiation oncologist, or other patientrelated factors. These limitations might have influenced our findings and will reduce the effectiveness of the existing clinical evidence. Indeed, more RCTs are needed to further support our conclusions.

\section{Conclusion}

In conclusion, we performed an up-to-date and comprehensive summary of the effect of HD-RT and SD-RT in ESCC patients who received modern radiotherapy and our results suggest that HD-RT (a higher radiation dose of $>=60 \mathrm{~Gy}$ ) could bring about better locoregional control and OS than SD-RT therapy. In the future, Phase III trials comparing the effect and toxicity of SD-RT and HD-RT using modern RT technique are warranted in the right subgroup population of ESCC patients.

\section{Supplementary information}

Supplementary information accompanies this paper at https://doi.org/10. 1186/s13014-019-1386-x.

Additional file 1. Summary of Finding Table.

\section{Abbreviations}

3DRT: Three dimensional conformal radiotherapy; CCRT: Definitive concurrent chemoradiotherapy; Cl: Confidence interval; CTV: Clinical target volume; CTVnd: Nodal clinical target volume; CTVt: Tumor clinical target volume EAC: Esophageal adenocarcinoma; EC: Esophageal cancer; ESCC: Esophageal squamous cell cancer; GTV: Gross tumor volume; GTVnd: Nodal gross tumor volume; HD-RT: High-dose radiotherapy; IMRT: Intensity-modulated radiotherapy; LC: Local control; LRFS: Local recurrence-free survival; OS: Overall survival; PFS: Progression-free survival; PTV: Planning target volume; SD-RT: Standard-dose radiotherapy

\section{Acknowledgments}

None

\section{Authors' contributions}

All authors read and approved the final manuscript prior to submission. HS Luo and HC Huang: conceived and designed the experiments; HC Huang and LX Lin: performed the experiments; HS Luo: analyzed the data; HS Luo: wrote the paper.

Funding

None.

Availability of data and materials

The datasets used and/or analyzed during the current study are available from the corresponding author upon reasonable request.

Ethics approval and consent to participate

Not applicable.

Consent for publication

Not applicable.

\section{Competing interests}

The authors declare that they have no competing interests.

Received: 4 August 2019 Accepted: 25 September 2019

Published online: 17 October 2019

References

1. Chen W, Zheng R, Baade PD, et al. Cancer statistics in China, 2015. CA Cancer J Clin. 2016;66(2):115-32.

2. Feng RM, Zong YN, Cao SM, et al. Current cancer situation in China: good or bad news from the 2018 global Cancer statistics? Cancer Commun (Lond). 2019;39(1):22

3. Zeng $H$, Zheng $R$, Zhang $S$, et al. Esophageal cancer statistics in China, 2011: estimates based on 177 cancer registries. Thorac Cancer. 2016;7(2):232-7.

4. Siegel R, Ma J, Zou Z, et al. Cancer statistics, 2014. CA Cancer J Clin. 2014; 64(1):9-29.

5. Cooper JS, Guo MD, Herskovic A, et al. Chemoradiotherapy of locally advanced esophageal cancer: long-term follow-up of a prospective randomized trial (RTOG 85-01). Radiation therapy oncology group. JAMA. 1999:281(17):1623-7.

6. Minsky BD, Pajak TF, Ginsberg RJ, et al. INT 0123 (radiation therapy oncology group 94-05) phase III trial of combined-modality therapy for esophageal cancer: high-dose versus standard-dose radiation therapy. J Clin Oncol. 2002;20(5):1167-74.

7. Li M, Zhang X, Zhao F, et al. Involved-field radiotherapy for esophageal squamous cell carcinoma: theory and practice. Radiat Oncol. 2016;11:18.

8. Song $T$, Liang $X$, Fang $M$, et al. High-dose versus conventional-dose irradiation in cisplatin-based definitive concurrent chemoradiotherapy for esophageal cancer: a systematic review and pooled analysis. Expert Rev Anticancer Ther. 2015;15(10):1157-69.

9. Fan CY, Su YF, Huang WY, et al. Definitive radiotherapy dose escalation with chemotherapy for treating non-metastatic oesophageal cancer. Sci Rep. 2018;8(1):12877.

10. Yu W, Cai XW, Liu Q, et al. Safety of dose escalation by simultaneous integrated boosting radiation dose within the primary tumor guided by (18)FDG-PET/CT for esophageal cancer. Radiother Oncol. 2015;114(2): 195-200.

11. Chen J, Guo H, Zhai T, et al. Radiation dose escalation by simultaneous modulated accelerated radiotherapy combined with chemotherapy for esophageal cancer: a phase II study. Oncotarget. 2016;7(16):22711-9.

12. Clavier JB, Antoni $D$, Atlani $D$, et al. Definitive chemoradiotherapy for esophageal cancer: 66Gy versus 50Gy, a retrospective study. Cancer Radiother. 2013;17(3):221-8. 
13. Suh YG, Lee IJ, Koom WS, et al. High-dose versus standard-dose radiotherapy with concurrent chemotherapy in stages II-III esophageal cancer. Jpn J Clin Oncol. 2014;44(6):534-40.

14. Chen CY, Li CC, Chien CR. Does higher radiation dose lead to better outcome for non-operated localized esophageal squamous cell carcinoma patients who received concurrent chemoradiotherapy? A population based propensity-score matched analysis. Radiother Oncol. 2016;120(1):136-9.

15. Kim HJ, Suh YG, Lee YC, et al. Dose-response relationship between radiation dose and loco-regional control in patients with stage II-III esophageal Cancer treated with definitive Chemoradiotherapy. Cancer Res Treat. 2017; 49(3):669-77.

16. Deng $\mathrm{Y}$, Bian $\mathrm{C}$, Tao $\mathrm{H}$, et al. Improved survival with higher radiation dose for esophageal squamous cell carcinoma patients treated with definitive chemoradiotherapy. Oncotarget. 2017;8(45):79662-9.

17. Chang CL, Tsai HC, Lin WC, et al. Dose escalation intensity-modulated radiotherapy-based concurrent chemoradiotherapy is effective for advanced-stage thoracic esophageal squamous cell carcinoma. Radiother Oncol. 2017;125(1):73-9.

18. Chen $H$, Zhou L, Yang $Y$, et al. Clinical effect of radiotherapy combined with chemotherapy for non-surgical treatment of the esophageal squamous cell carcinoma. Med Sci Monit. 2018;24:4183-91.

19. Fletcher GH. Clinical dose-response curves of human malignant epithelial tumours. Br J Radiol. 1973;46(541):1-12.

20. Tong DK, Law S, Kwong DL, et al. Histological regression of squamous esophageal carcinoma assessed by percentage of residual viable cells after neoadjuvant chemoradiation is an important prognostic factor. Ann Surg Oncol. 2010;17(8):2184-92.

21. Chen Y, Zhu HP, Wang T, et al. What is the optimal radiation dose for nonoperable esophageal cancer? Dissecting the evidence in a meta-analysis. Oncotarget. 2017;8(51):89095-107.

22. Yamashita H, Nakagawa K, Tago M, et al. The intergroup/RTOG 85-01 concurrent chemoradiation regimen for Japanese esophageal cancer. Hepatogastroenterology. 2006;53(72):863-8.

23. Ling TC, Slater JM, Nookala P, et al. Analysis of intensity-modulated radiation therapy (IMRT), proton and 3D conformal radiotherapy (3D-CRT) for reducing perioperative cardiopulmonary complications in esophageal Cancer patients. Cancers (Basel). 2014:6(4):2356-68.

24. Suntharalingam $M$, Winter $K$, Ilson $D$, et al. Effect of the addition of Cetuximab to paclitaxel, Cisplatin, and radiation therapy for patients with esophageal Cancer: the NRG oncology RTOG 0436 phase 3 randomized clinical trial. JAMA Oncol. 2017;3(11):1520-8.

25. Hsieh HY, Hsu CP, Yeh HL, et al. Definite intensity-modulated radiotherapy with concurrent chemotherapy more than 4 cycles improved survival for patients with locally-advanced or inoperable esophageal squamous cell carcinoma. Kaohsiung J Med Sci. 2018;34(5):281-9.

26. Zhang W, Luo Y, Wang $X$, et al. Dose-escalated radiotherapy improved survival for esophageal cancer patients with a clinical complete response after standard-dose radiotherapy with concurrent chemotherapy. Cancer Manag Res. 2018;10:2675-82.

27. Brower JV, Chen S, Bassetti MF, et al. Radiation dose escalation in esophageal Cancer revisited: a contemporary analysis of the National Cancer Data Base, 2004 to 2012. Int J Radiat Oncol Biol Phys. 2016;96(5): 985-93.

28. He L, Allen PK, Potter A, et al. Re-evaluating the optimal radiation dose for definitive chemoradiotherapy for esophageal squamous cell carcinoma. J Thorac Oncol. 2014;9(9):1398-405.

\section{Publisher's Note}

Springer Nature remains neutral with regard to jurisdictional claims in published maps and institutional affiliations.

Ready to submit your research? Choose BMC and benefit from:

- fast, convenient online submission

- thorough peer review by experienced researchers in your field

- rapid publication on acceptance

- support for research data, including large and complex data types

- gold Open Access which fosters wider collaboration and increased citations

- maximum visibility for your research: over $100 \mathrm{M}$ website views per year

At $\mathrm{BMC}$, research is always in progress.

Learn more biomedcentral.com/submissions 